\title{
Prevalence of Metabolic Syndrome and Association with Grip Strength in Older Adults: Findings from the HOPE Study
}

This article was published in the following Dove Press journal:

Diabetes, Metabolic Syndrome and Obesity: Targets and Therapy

\author{
Reshma Aziz Merchant (D) ${ }^{1,2}$ \\ Yiong Huak Chan ${ }^{3}$ \\ Jia Yi Lim² \\ John E Morley ${ }^{4}$ \\ 'Division of Geriatric Medicine, \\ Department of Medicine, National \\ University Hospital, Singapore; \\ ${ }^{2}$ Department of Medicine, Yong Loo Lin \\ School of Medicine, National University \\ of Singapore, Singapore; ${ }^{3}$ Biostatistics \\ Unit, Yong Loo Lin School of Medicine, \\ National University of Singapore, \\ Singapore; ${ }^{4}$ Division of Geriatric \\ Medicine, Saint Louis University School of \\ Medicine, St Louis, MO, USA
}

Correspondence: Reshma Aziz Merchant Division of Geriatric Medicine, Department of Medicine, National University Hospital II 9228, Singapore Tel +65 67795555

Email reshmaa@nuhs.edu.sg
Objective: To determine the prevalence of metabolic syndrome (MetS) in older adults and assess the association of MetS and adverse outcomes with handgrip strength (HGS), HGS/ body weight (BWT), and HGS/body mass index (BMI).

Methods: A cross-sectional population study in Singapore. Data were collected on demographics, HGS, Timed-Up and Go (TUG), fasting glucose, lipid profile, blood pressure, waist circumference, frailty status, and cognition in 722 older adults $\geq 65$ years old. MetS was defined using the Modified ATP III for Asians where at least three of the following conditions must be fulfilled, central obesity, high blood glucose (or diagnosed diabetes mellitus), high blood pressure (or diagnosed hypertension), low high-density lipoprotein, and high triglycerides. The waist circumference in the Modified ATP III for Asians is $\geq 90 \mathrm{~cm}$ for males or $\geq 80 \mathrm{~cm}$ for females. HGS and HGS normalized by BWT or BMI were used for the association.

Results: The prevalence of MetS in older adults was $41.0 \%$, and those $\geq 85$ years old $50.0 \%$. The prevalence was higher in females $\geq 70$ years old, with 8 in 10 females $\geq 85$ years having MetS. After adjusting for age, years of education, physical exercise, as well as history of smoking and alcohol consumption, higher HGS normalized by BWT or BMI was significantly associated with lower odds of having MetS (OR: $0.51,95 \%$ CI $0.43-0.61, p<0.01)$ and (OR: $0.13,95 \%$ CI $0.07-0.24, p<0.01$ ).

Conclusion: Almost 1 in 2 older adults had MetS, with the prevalence in females much higher than that in males over 70 years old. Our findings suggest that both HGS/BWT and HGS/BMI had a significant negative association with MetS, its components, and adverse effects. Further studies are needed to validate the association and to determine optimal cutoffs of HGS/BWT and HGS/BMI for MetS, and the effectiveness of interventions in averting the risk.

Keywords: handgrip strength, metabolic syndrome, prevalence, sarcopenia, older adults

\section{Introduction}

The prevalence of metabolic syndrome (MetS) is rapidly increasing in countries with fast-aging populations. ${ }^{1}$ MetS is defined as a cluster of cardiometabolic risk factors including diabetes, central obesity, hypertension, elevated fasting plasma glucose, elevated triglycerides, and reduced high-density lipoprotein. It is associated with increased morbidity and mortality including non-alcoholic fatty liver disease, cognitive impairment, cardiovascular disease, and functional decline. ${ }^{2,3}$ Skeletal muscle plays a crucial role in the body's glucose metabolism and up to three-quarters of $\geq 75$-year-old people have glucose intolerance. ${ }^{4}$ Insulin resistance is increasingly known as a major 
cause of MetS. ${ }^{5}$ Several studies have reported that sarcopenia and low muscle mass are risk factors for MetS. ${ }^{6-8}$ The current consensus on the diagnosis of sarcopenia includes low muscle strength, low skeletal muscle mass, and/or low physical performance. Handgrip strength (HGS) correlates with total muscle strength and has been shown to predict many adverse outcomes including functional decline, falls, and mortality. ${ }^{9-11}$ While there is an association between HGS and MetS, a direct correlation between HGS and MetS is still an ongoing debate. ${ }^{12-14}$ It was initially proposed by PloutzSnyder et al that correcting muscle strength for bodyweight was a better predictor of functional performance, and more recent findings reported that HGS adjusted for BMI or BWT may better reflect muscle quality. ${ }^{13,15}$ Muscle power is thought to decline before muscle strength followed by muscle mass, and is relevant for many daily tasks which in turn could be attributed to muscle quality. ${ }^{16}$

In countries with rapidly aging populations, screening for risk factors, detection, and prevention should be an urgent priority. There are recent studies suggesting HGS/ BMI or relative grip strength is better correlated with MetS and adverse outcomes. In addition, to our knowledge, there are no studies on the prevalence of MetS in older adults in Singapore. The aim of our study was to determine the prevalence of MetS in older adults, and assess the association of MetS, components of MetS, and adverse outcomes with HGS, HGS/BWT, and HGS/BMI.

\section{Methods}

\section{Participants}

The population of this study is a subgroup of the Healthy Older People Everyday (HOPE) study, a sub-cohort of the Singapore Population Health Studies - Community Health Study, conducted between April 2015 and August 2016. ${ }^{17}$ HOPE was a cross-sectional and nationally representable cohort where 1051 older adults $\geq 65$ years old were recruited from a defined geographical area in the northwest of Singapore. Participants were excluded if baseline information on MetS was incomplete or participants did not agree on fasting blood tests. Complete data were available for only 722 participants. Further details on recruitment, questionnaires, physical and cognitive assessment are elaborated in prior publications. ${ }^{17,18}$

\section{Procedures}

The demographic questionnaire included questions on socio-demographics, lifestyle, education level, chronic illness, falls, physical activity including the number of minutes walked or cycled each day, physical and mental health, quality of life, and functional status, which were self-reported by the participants. Mental health was assessed using Mini Mental State Exam (MMSE) and cognitive impairment was defined as MMSE $<24 .{ }^{19}$ The cognitive level was further adjusted using age and education level. ${ }^{20}$ Katz Index for activities of daily living (ADL) was administered to assess participants' functioning level. ${ }^{21}$ Participants reported their level of independence in performing six different functions, namely, bathing, dressing, toileting, transferring, and continence. The 5-item FRAIL scale (Fatigue, Resistance, Ambulation, Illness, and Loss of Weight) which has been validated in many countries was used to screen for frailty. ${ }^{22,23}$ A score of 0 represents robust, 1-2 represents pre-frail, and 3-5 represents frail. Selfperceived health rating was assessed using the EQ-Visual Analogue Scale (VAS) where participants rate their overall health from a scale of 0 (poorest state of health) to 100 (best state of health) by looking at a $20 \mathrm{~cm}$ long scale. ${ }^{24}$ These were all conducted during a face-to-face interview at the participant's home or the nearby Resident's Committee center.

\section{Physical and Biochemical Assessment}

Following the interview, participants were given an option to undergo a health screening which comprised of physical examination and blood test. The physical examination included measurement of weight, height, blood pressure, and waist circumference. Waist circumference (WC) was measured midpoint between last rib and the iliac crest, blood pressure measured in seating position and the average of two attempts was used. Physical performance screening included the Timed-Up-and-Go (TUG) $\operatorname{test}^{25}$ and HGS. TUG recorded the time the participant took to raise up from a chair, walk a distance of $3 \mathrm{~m}$ and return back to the seat using a stopwatch. HGS was measured three times in a seated position with each arm flexed at a $90^{\circ}$ angle using a digital dynamometer (A5401, Takei Scientific Instruments Co., Ltd, Japan). Only the maximum HGS reading for the dominant hand was used in analyses together with HGS normalized with BWT or BMI. Fasting bloods were collected in the morning for lipid profiling which included triglycerides (TG), high-density lipoprotein cholesterol (HDLc), and glucose. Collected blood samples were analyzed on the same day at National University Hospital laboratories. 


\section{Metabolic Syndrome}

The definition of MetS was based on criteria defined by the Modified ATP III for Asians which includes the presence of 3 or more of the following five components 1) waist circumference $\geq 90 \mathrm{~cm}$ for males or $\geq 80 \mathrm{~cm}$ for females, 2) TG $\geq 150 \mathrm{mg} / \mathrm{dL}, 3) \mathrm{HDLc}<40 \mathrm{mg} / \mathrm{dL}$ in males or $<50 \mathrm{mg} / \mathrm{dL}$ in females, 4) blood pressure (BP) $\geq 130 / 85 \mathrm{mmHg}$ or use of antihypertensive medication, and 5) fasting plasma glucose $\geq 100 \mathrm{mg} / \mathrm{dL}$ or use of pharmacologic treatment for diabetes mellitus. $^{26}$

\section{Statistical Analysis}

All analyses were performed using IBM SPSS Statistics 25.0 with statistical significance set at $p<0.05$. Characteristics of participants were presented as mean and standard deviation for continuous variables while categorical variables were presented as frequencies and percentages. Differences in the numerical variables between those with and without MetS were assessed using 2 sample $t$-test when normality and homogeneity assumptions were satisfied, otherwise Mann-Whitney $U$-test was performed. Chi-square test was used for categorical variables. Binary logistic regression was performed to determine the influence of HGS, HGS/BWT, and HGS/ BMI on MetS, its individual components, ADL impairment, frailty status, and cognitive impairment. The z-scores of HGS, HGS/BWT, and HGS/BMI were used to obtain a standardized comparison of the odds ratios on MetS. All logistics models had the Hosmer Lemeshow goodness of fit statistic satisfied except for at least prefrail, thus results need to be interpreted with caution. Linear regression was performed to determine the influence of the same predictors on TUG. Regression models were unadjusted in Model 1 and adjusted for age in Model 2 as well as years of education, walking/cycling duration, history of smoking and alcohol consumption in Model 3. The assumptions of constant variance and residual normality were checked.

The study protocol was approved by the National Healthcare Group (NHG) Domain Specific Review Board (DSRB) whose requirements are based on the Declaration of Helsinki and the ethical principles in the Belmont Report, and all participants signed an informed consent form. Any research proposals which involve the National University of Singapore and other restructured hospitals in Singapore can be submitted to NHG DSRB.

\section{Results}

The prevalence of MetS in the sampled population was $41.0 \%$. Table 1 presents the demographic differences between MetS and no-MetS older adults. There was no significant age or gender difference between the two groups. There were significant ethnic differences in the prevalence of MetS with one in two of the Indian ethnic group, compared to one in three amongst the Chinese ethnic group. The MetS group had significantly higher BMI (26.5 $\pm 3.8 \mathrm{~kg} / \mathrm{m}^{2}$ vs $\left.23.0 \pm 3.5 \mathrm{~kg} / \mathrm{m}^{2}\right)$ and higher systolic blood pressure $(138 \pm 20 \mathrm{mmHg}$ vs $131 \pm 19 \mathrm{mmHg})$ than the noMetS group. The MetS group was also less active, on average walking or cycling for $34 \pm 74$ minutes compared with no-MetS $40 \pm 50$ minutes. The prevalence of frailty amongst the MetS group was almost double $(7.2 \%$ vs $4.5 \%$ ), with almost 1 in 7 having difficulty walking 50 metres and 1 in 10 having more than 5 illnesses. Although non-significant, there were more older adults with MetS who were separated/divorced or widowed and had lower education.

Those with MetS had significantly longer TUG of $11.9 \pm$ 3.7 compared with $10.8 \pm 4.1$ seconds and worse cognition based on the MMSE score $(26.2 \pm 4.5$ vs $27.2 \pm 3.1)$. Though not statistically significant, maximum HGS was lower, and after normalizing for BWT and BMI, respectively, significant differences between the groups were observed.

Figure 1 shows the prevalence of MetS across gender and the 5-year age groups. The overall prevalence increased from 65 to 74 years old from $40.5 \%$ to $44.7 \%$, reduced to $32.0 \%$ between 75 and 79 years, and increased again to $50 \%$ for the $\geq 85$-year-old participants with MetS. The prevalence of MetS amongst females was higher than for males $\geq 70$ years old, although the confidence interval overlapped, with 8 in 10 females $\geq 85$ years having MetS.

Figure 2 shows the prevalence of individual MetS components across age, with hypertension being most prevalent across gender and age groups. As age increased, prevalence of hypertension was observed to increase. Generally, more females had central obesity as compared to men, regardless of age groups, and prevalence increased with age. The prevalence of elevated fasting glucose and/ or diabetes remained relatively stable between $25 \%$ and $45 \%$ across gender and age group. A slightly lower proportion of females had high TG as compared to males below 75 years old, where the trend reversed after the age of 75 years with a greater difference in prevalence with increasing age. The prevalence of low HDL in men 
Table I Characteristics of Participants in Mean \pm Standard Deviation or Frequency (Percentage) in 722 Elderly in the HOPE Study (2015-2016)

\begin{tabular}{|c|c|c|c|c|}
\hline & $\begin{array}{l}\text { All } \\
722(100.0)\end{array}$ & $\begin{array}{l}\text { No Metabolic Syndrome } \\
426(59.0)\end{array}$ & $\begin{array}{l}\text { Has Metabolic Ssyndrome } \\
296(41.0)\end{array}$ & P-value \\
\hline Age, years & $71 \pm 5$ & $71 \pm 5$ & $71 \pm 5$ & 0.970 \\
\hline Gender,n & & & & 0.119 \\
\hline Male & $325(45.0)$ & $202(47.4)$ & $123(41.6)$ & \\
\hline Female & $397(55.0)$ & $224(52.6)$ & $173(58.4)$ & \\
\hline Ethnicity, $n^{\wedge}$ & & & & 0.017 \\
\hline Chinese & $592(82.0)$ & $365(61.7)$ & $227(38.3)$ & \\
\hline Malay & $4 \mathrm{I}(5.7)$ & $19(46.3)$ & $22(53.7)$ & \\
\hline Indian & $44(6.1)$ & $19(43.2)$ & $25(56.8)$ & \\
\hline Body weight, kg & $61.3 \pm 11.0$ & $57.9 \pm 9.9$ & $66.3 \pm 10.7$ & $<0.01$ \\
\hline $\mathrm{BMI}, \mathrm{kg} / \mathrm{m}^{2}$ & $23.4 \pm 4.1$ & $23.0 \pm 3.5$ & $26.5 \pm 3.8$ & $<0.01$ \\
\hline Education, years & $6.3 \pm 4.4$ & $6.5 \pm 4.4$ & $6.0 \pm 4.3$ & 0.133 \\
\hline Systolic pressure, $\mathrm{mmHg}$ & $134 \pm 20$ & $|3| \pm 19$ & $138 \pm 20$ & $<0.01$ \\
\hline Diastolic pressure, $\mathrm{mmHg}$ & $70 \pm 10$ & $69 \pm 9$ & $71 \pm 10$ & $<0.01$ \\
\hline \multicolumn{5}{|l|}{ Physical activity, n } \\
\hline Engage in vigorous activity & $26(3.6)$ & $18(4.2)$ & $8(2.7)$ & 0.280 \\
\hline Engage in moderate activity & $221(30.6)$ & $136(31.9)$ & $85(28.7)$ & 0.358 \\
\hline Walk/bike per day (mins) & $38 \pm 59$ & $40 \pm 51$ & $36 \pm 69$ & 0.191 \\
\hline History of smoking,n & $212(29.4)$ & $123(28.9)$ & $89(30.1)$ & 0.729 \\
\hline History of alcohol consumption,n & $421(58.3)$ & $264(62.0)$ & $157(53.0)$ & 0.017 \\
\hline \multicolumn{5}{|l|}{ MetS components, $n$} \\
\hline Central obesity & $436(60.4)$ & $168(39.4)$ & $269(90.5)$ & $<0.01$ \\
\hline Hypertension & $538(74.5)$ & $264(62.0)$ & $274(92.6)$ & $<0.01$ \\
\hline Elevated fasting glucose & $223(30.9)$ & $48(11.3)$ & $175(59.1)$ & $<0.01$ \\
\hline High triglycerides & $193(26.7)$ & $34(8.0)$ & $159(53.7)$ & $<0.01$ \\
\hline Low high-density lipoprotein & $219(30.3)$ & $48(11.3)$ & $17 \mid(57.8)$ & $<0.01$ \\
\hline Frail status, $\mathrm{n}$ & & & & 0.015 \\
\hline Robust & $420(58.6)$ & $267(62.8)$ & $153(52.4)$ & \\
\hline Pre-frail & $257(35.8)$ & $139(32.7)$ & $118(40.4)$ & \\
\hline Frail & $40(5.6)$ & $19(4.5)$ & $21(7.2)$ & \\
\hline Fatigue, $n$ & $182(25.4)$ & $97(22.8)$ & $85(29.1)$ & 0.057 \\
\hline Resistance, $n$ & 77 (10.7) & $4 \mid(9.6)$ & $36(12.3)$ & 0.254 \\
\hline Aerobic, $\mathrm{n}$ & $82(11.4)$ & $41(9.6)$ & $4 \mid(14.0)$ & 0.069 \\
\hline Illnesses, n & $34(4.7)$ & $10(2.4)$ & $24(8.2)$ & $<0.01$ \\
\hline Loss Of Weight, $n$ & $83(11.6)$ & $46(10.8)$ & $37(12.7)$ & 0.447 \\
\hline At least one $A D L$ impairment, $n$ & $118(16.3)$ & $59(13.8)$ & $59(19.9)$ & 0.030 \\
\hline One or more falls, $n$ & $86(11.9)$ & $48(11.3)$ & $38(12.8)$ & 0.522 \\
\hline Timed-up and go, $s$ & $11.3 \pm 4.0$ & $10.8 \pm 4.1$ & $11.9 \pm 3.7$ & $<0.01$ \\
\hline Grip strength, kg & $22.5 \pm 6.9$ & $22.8 \pm 6.7$ & $22.1 \pm 7.1$ & 0.165 \\
\hline Grip strength/weight & $0.4 \pm 0.1$ & $0.4 \pm 0.1$ & $0.3 \pm 0.1$ & $<0.01$ \\
\hline Grip strength/BMI & $1.0 \pm 0.3$ & $1.0 \pm 0.3$ & $0.9 \pm 0.3$ & $<0.01$ \\
\hline Health rating, score & $80.8 \pm 14.4$ & $81.4 \pm 14.0$ & $80.1 \pm 14.9$ & 0.294 \\
\hline MMSE, score & $26.8 \pm 3.7$ & $27.2 \pm 3.1$ & $26.2 \pm 4.5$ & $<0.01$ \\
\hline Poor cognition (MMSE 0-23), $n$ & $64(8.9)$ & $33(7.7)$ & $31(10.5)$ & 0.205 \\
\hline Poor cognition by age and education, $n$ & $45(6.2)$ & $21(4.9)$ & $24(8.1)$ & 0.082 \\
\hline
\end{tabular}

Notes: Figures presented as means \pm SD for continuous variables or frequencies (\%) for categorical variables. P-value for continuous variables (Mann-Whitney $U$-test) and for categorical variables (Chi-square). ^Percentages within ethnic group.

Abbreviations: BMI, body mass index; ADL, activities of daily living; MMSE, mini-mental state examination. 


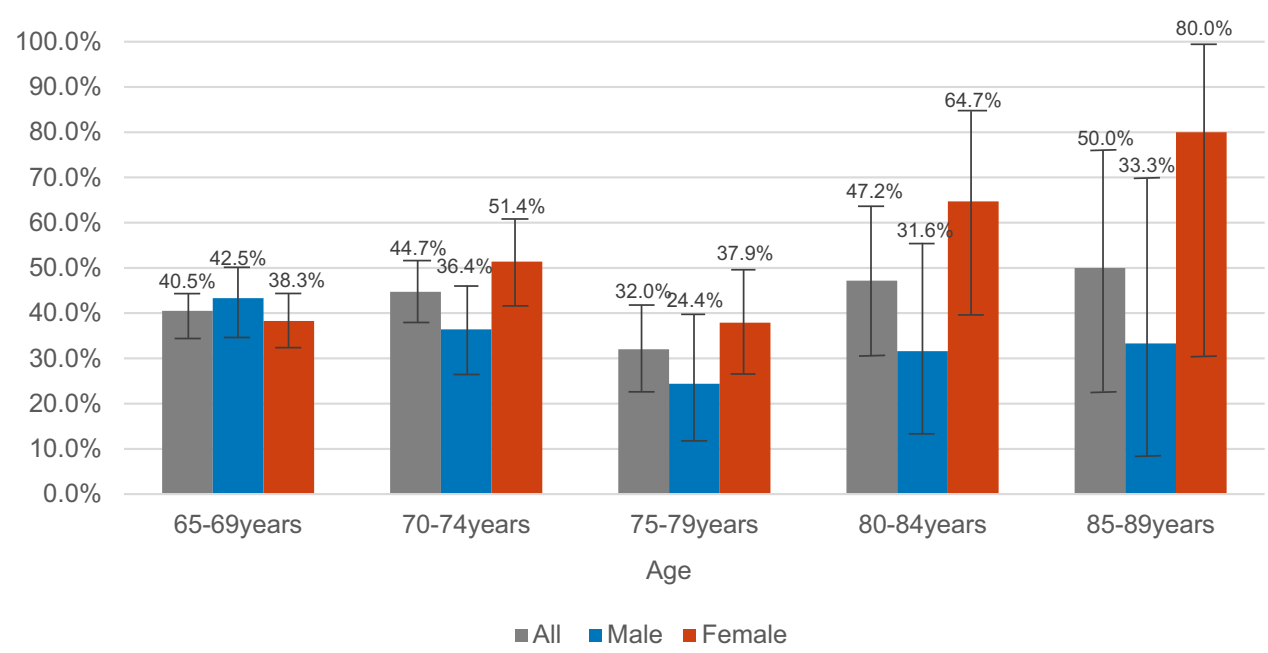

Figure I Prevalence of MetS by gender and age groups.

remained between $15 \%$ and $30 \%$, while that of females was higher, especially above 80 years old.

The odds ratios of individual HGS indices on the risk of MetS are summarized in Table 2. Only after normalizing for BWT and BMI, respectively, HGS was observed to be associated with decreased odds of having MetS by almost $50 \%$ and $90 \%$, respectively (OR $0.51,95 \%$ CI $0.43-0.61, p<0.01$, and OR $0.13,95 \%$ CI $0.07-0.24, p<0.01)$. Similar associations were also found for HGS/BWT and HGS/BMI on all other components of MetS. Additionally, the unadjusted model using $\mathrm{Z}$ scores showed that the measure of grip strength alone was associated with a reduction in risk of having MetS by $17 \%$ as compared to a reduction of $34 \%$ and $32 \%$ in risk associated with HGS/BWT and HGS/BMI, respectively. This suggests that the normalized grip measures were twice as effective as grip strength in predicting a reduction in risk of MetS. Similarly, HGS/BWT and HGS/BMI better predicted adverse outcomes compared with HGS alone for cognitive impairment, frailty/pre-frailty, TUG, and ADL impairment.

\section{Discussion}

The prevalence of MetS varies depending on the criteria used and age, and the worldwide prevalence of MetS is known to be between $22 \%$ and $44 \% .{ }^{27}$ The prevalence of MetS in our population increased up to the age of 70 years, took a sharp decline, and increased again. One of the hypotheses is that a subgroup with very high-risk cardiovascular risk factors may die, and the subsequent increase may be related to a sedentary lifestyle, mobility limitation, and lack of physical activity. ${ }^{28-30}$ The participants in the MetS group walked or cycled less. A combination of health education and walking exercise has been shown to reduce risk factors for MetS and lead to an improved lifestyle. ${ }^{31}$ Given that prevalence of MetS increases with age, and almost all of the subjects had high waist circumference, efforts to increase awareness must begin early, and should be a public health priority for every country. Studies have shown that exercise can reduce or improve most parameters associated with MetS. ${ }^{32}$ The higher prevalence of MetS in females after the age of 70 years is linked with menopause, where females with 20 years or more since menopause are more likely to have MetS and elevated blood pressure compared with those for whom menopause was 10 years ago or less. $^{33}$ The changes in lipid profile including increasing TG, total cholesterol, and low HDL levels are known to occur during the menopausal transition and post-menopause. ${ }^{34}$ An interesting aspect of our study was that the prevalence of low HDL almost doubled in females after the age of 80 years old.

In addition to low relative HGS, our MetS group also had longer TUG. In a most recent study, longer TUG with or without low HGS was associated with increased short-term mortality. ${ }^{35}$ Similar to other studies, our MetS group had significantly lower MMSE scores and a higher prevalence of cognitive impairment. ${ }^{36}$ Persons with MetS are more likely to have vascular dementia related to hypertension, elevated fasting glucose, and hypertriglyceridemia. Similarly, frail persons are more likely to have elevated fasting glucose, and have a higher prevalence of cognitive impairment too. ${ }^{37-40}$

Of the MetS components, obesity and insulin resistance are known to be associated with sarcopenia. Sarcopenia and insulin resistance share a common pathway where the loss of skeletal muscle mass gives rise to insulin resistance, impaired suppression of gluconeogenesis, and reduced protein 
A

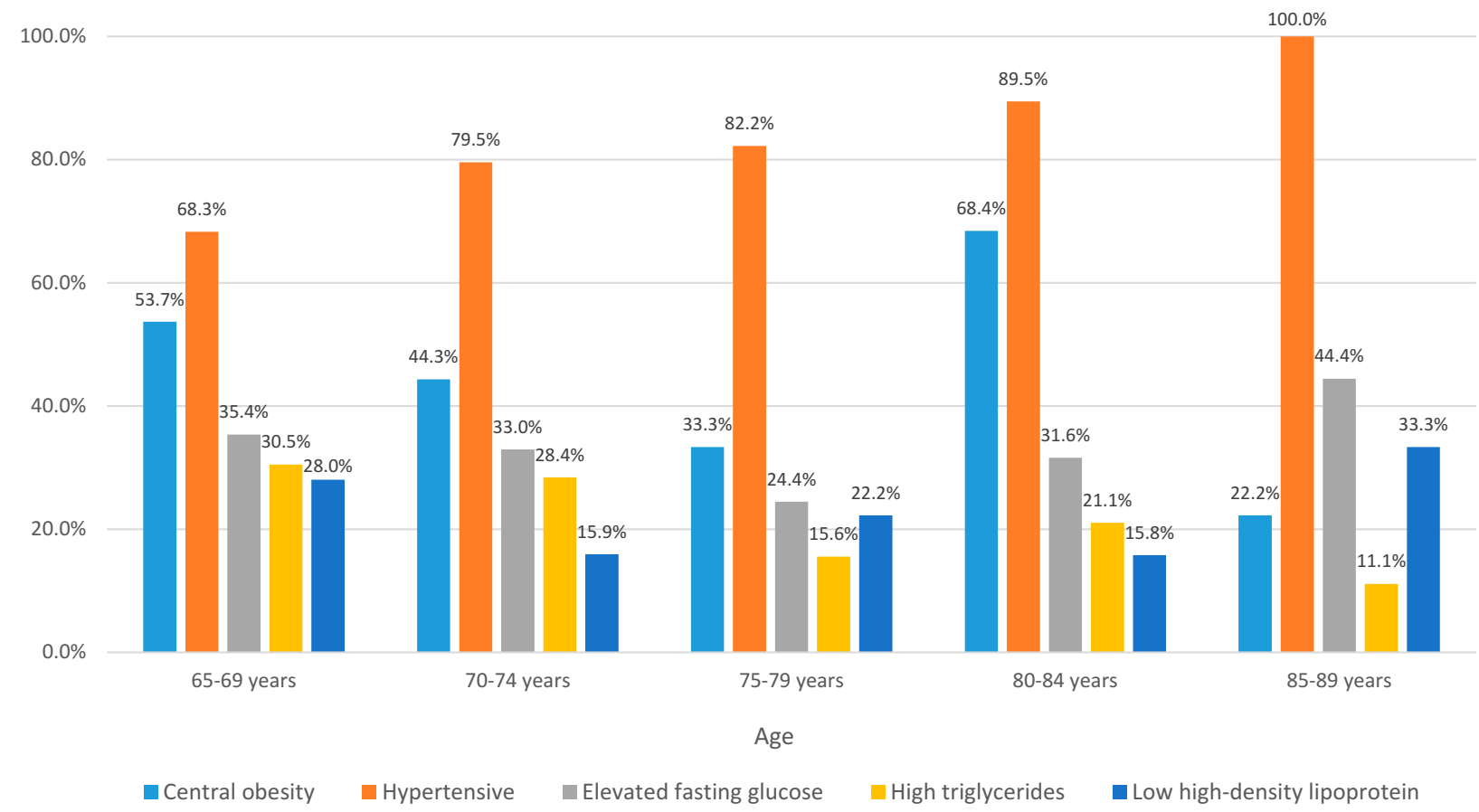

B

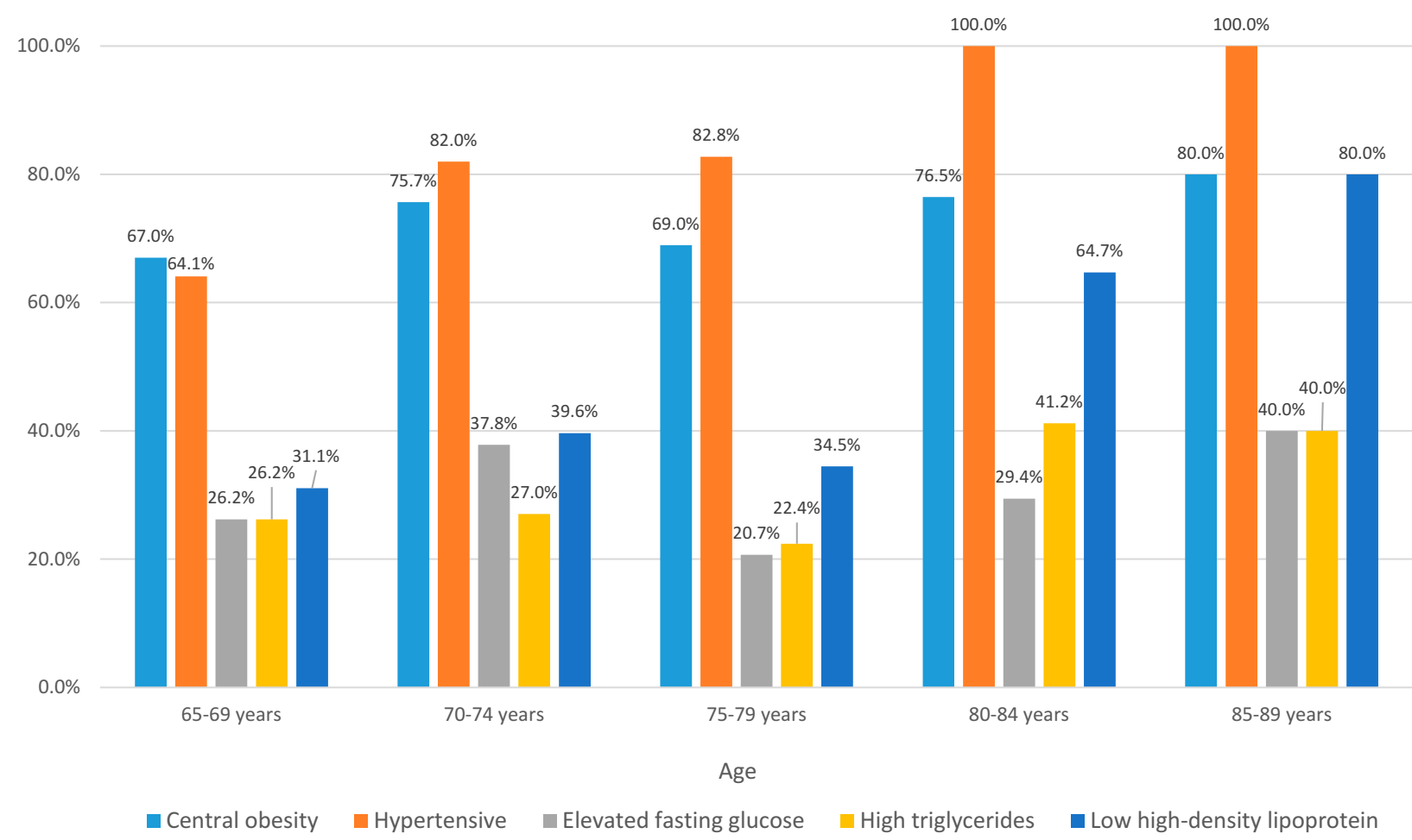

Figure 2 (A) Prevalence of MetS components across age groups (for males). (B) Prevalence of MetS components across age groups (for females). 
Table 2 Odds Ratio and Beta-Coefficients (95\% Confidence Interval) of Individual Grip Strength Indices on Risk of Metabolic Syndrome, Its Components, Cognition, and Physical Decline in 722 Older adults in the HOPE Study (2015-2016)

\begin{tabular}{|c|c|c|c|}
\hline & $\begin{array}{l}\text { Model I } \\
\text { OR/Beta-Coefficient } \\
(95 \% \mathrm{Cl})\end{array}$ & $\begin{array}{l}\text { Model } 2 \\
\text { OR/Beta-Coefficient } \\
(95 \% \mathrm{Cl})\end{array}$ & $\begin{array}{l}\text { Model } 3 \\
\text { OR/Beta-Coefficient } \\
(95 \% \mathrm{Cl})\end{array}$ \\
\hline \multicolumn{4}{|l|}{ Grip strength } \\
\hline Metabolic syndrome & $0.99(0.97-1.01)$ & $0.99(0.96-1.01)$ & $0.99(0.96-1.01)$ \\
\hline Central obesity & $0.95(0.93-0.97)^{* *}$ & $0.95(0.93-0.97)^{* *}$ & $0.96(0.94-0.99)^{* *}$ \\
\hline Hypertension & $0.99(0.97-1.02)$ & $1.01(0.98-1.03)$ & $1.02(0.99-1.05)$ \\
\hline Elevated fasting glucose & $0.99(0.97-1.01)$ & $0.99(0.97-1.01)$ & $0.98(0.96-1.01)$ \\
\hline High triglycerides & $1.01(0.99-1.03)$ & $1.01(0.98-1.03)$ & $1.01(0.98-1.03)$ \\
\hline Low high-density lipoprotein & $0.97(0.94-0.99)^{* *}$ & $0.97(0.95-0.99)^{* *}$ & $0.98(0.96-1.0 \mathrm{I})$ \\
\hline Poor cognition (MMSE 0-23) & $0.89(0.85-0.93)^{* *}$ & $0.89(0.85-0.94)^{* *}$ & $0.90(0.85-0.95)^{* *}$ \\
\hline Poor cognition adjusted by age and education & $0.85(0.80-0.90)^{* *}$ & $1.03(1.02-1.06)^{* *}$ & $0.86(0.80-0.92)^{* *}$ \\
\hline At least I ADL impairment & $0.95(0.92-0.98)^{* *}$ & $0.86(0.80-0.91)^{* *}$ & $0.96(0.93-1.00)^{*}$ \\
\hline Pre-frail or frail & $0.97(0.95-0.99)^{* *}$ & $0.97(0.95-1.00)^{*}$ & $0.98(0.95-1.01)$ \\
\hline Timed up and go ${ }^{\#}$ & $-0.15(-0.20--0.11)^{* *}$ & $-0.12(-0.16-0.08)^{* *}$ & $-0.13(-0.18-0.09)^{* *}$ \\
\hline \multicolumn{4}{|l|}{ Grip strength/body weight } \\
\hline Metabolic syndrome & $0.55(0.47-0.64)^{* *}$ & $0.54(0.46-0.64)^{* *}$ & $0.5 \mathrm{I}(0.43-0.6 \mathrm{I})^{* *}$ \\
\hline Central obesity & $0.30(0.24-0.36)^{* *}$ & $0.29(0.23-0.35)^{* *}$ & $0.28(0.22-0.35)^{* *}$ \\
\hline Hypertension & $0.72(0.6 \mathrm{I}-0.84)^{* *}$ & $0.75(0.64-0.88)^{* *}$ & $0.75(0.63-0.90)^{* *}$ \\
\hline Elevated fasting glucose & $0.74(0.64-0.87)^{* *}$ & $0.74(0.63-0.86)^{* *}$ & $0.71(0.59-0.85)^{* *}$ \\
\hline High triglycerides & $0.81(0.69-0.95)^{* *}$ & $0.80(0.68-0.94)^{* *}$ & $0.76(0.64-0.90)^{* *}$ \\
\hline Low high-density lipoprotein & $0.69(0.59-0.81)^{* *}$ & $0.69(0.59-0.81)^{* *}$ & $0.73(0.61-0.86)^{* *}$ \\
\hline Poor cognition (MMSE 0-23) & $0.52(0.39-0.69)^{* *}$ & $0.53(0.40-0.71)^{* *}$ & $0.58(0.43-0.80)^{* *}$ \\
\hline Poor cognition adjusted by age and education & $1.08(0.94-1.25)$ & $0.44(0.31-0.63)^{* *}$ & $0.50(0.34-0.73)^{* *}$ \\
\hline At least I ADL impairment & $0.43(0.30-0.61)^{* *}$ & $0.75(0.6 I-0.92)^{* *}$ & $0.77(0.62-0.96)^{*}$ \\
\hline Pre-frail or frail & $0.78(0.68-0.90)^{* *}$ & $0.80(0.69-0.92)^{* *}$ & $0.83(0.7 \mathrm{I}-0.97)^{*}$ \\
\hline Timed up and go & $-1.02(-1.29--0.77)^{* *}$ & $-0.88(-1.11--0.64)^{* *}$ & $-0.90(-1.16--0.65)^{* *}$ \\
\hline \multicolumn{4}{|l|}{ Grip strength/BMI } \\
\hline Metabolic syndrome & $0.19(0.1 \mid-0.31)^{* *}$ & $0.19(0.11-0.31)^{* *}$ & $0.13(0.07-0.24)^{* *}$ \\
\hline Central obesity & $0.03(0.02-0.06)^{* *}$ & $0.03(0.02-0.05)^{* *}$ & $0.02(0.01-0.04)^{* *}$ \\
\hline Hypertension & $0.35(0.21-0.57)^{* *}$ & $0.40(0.24-0.67)^{* *}$ & $0.38(0.21-0.69)^{* *}$ \\
\hline Elevated fasting glucose & $0.50(0.30-0.82)^{* *}$ & $0.49(0.30-0.81)^{* *}$ & $0.39(0.22-0.7 \mathrm{I})^{* *}$ \\
\hline High triglycerides & $0.57(0.37-0.95)^{*}$ & $0.54(0.32-0.92)^{*}$ & $0.43(0.23-0.78)^{* *}$ \\
\hline Low high-density lipoprotein & $0.28(0.17-0.48)^{* *}$ & $0.29(0.17-0.49)^{* *}$ & $0.34(0.18-0.6 \mathrm{I})^{* *}$ \\
\hline Poor cognition (MMSE 0-23) & $0.06(0.02-0.17)^{* *}$ & $0.07(0.02-0.19)^{* *}$ & $0.08(0.03-0.28)^{* *}$ \\
\hline Poor cognition adjusted by age and education & $0.03(0.01-0.12)^{* *}$ & $0.03(0.01-0.13)^{* *}$ & $0.05(0.01-0.20)^{* *}$ \\
\hline At least I ADL impairment & $0.37(0.19-0.70)^{* *}$ & $0.42(0.21-0.82)^{*}$ & $0.42(0.20-0.90)^{*}$ \\
\hline Pre-frail or frail & $0.49(0.31-0.78) * *$ & $0.53(0.33-0.85)^{* *}$ & $0.62(0.36-1.06)$ \\
\hline Timed up and go & $-3.26(-4.12--2.40)^{* *}$ & $-2.77(-3.56--1.99)^{* *}$ & $-3.02(-3.91--2.14)^{* *}$ \\
\hline
\end{tabular}

Notes: Model I - Unadjusted, Model 2 - Adjusted for age, Model 3 - Adjusted for age, years of education, duration of exercise, history of smoking and alcohol consumption. ${ }^{*} p<0.05, *^{*} p<0.01$, ${ }^{*}$ Not standardized coefficient.

Abbreviations: MMSE, mini-mental state examination; ADL, activities of daily living; BMI, body mass index.

synthesis. Diabetes and insulin resistance are associated with a decline in skeletal muscle mass. ${ }^{41}$ Mitochondrial dysfunction which is prevalent in obesity is also associated with other age-related diseases including sarcopenia, cancers, and neurodegenerative disease. ${ }^{42}$ Low muscle mass is a known cause for the development of MetS. ${ }^{6,7,43}$ MetS has been shown to be positively associated with appendicular lean mass and forearm cross-sectional area and negatively correlated with muscle quality, especially in obese and overweight older adults with MetS. $^{44}$ There is a positive feedback loop between MetS and sarcopenia, where sarcopenia may also be exacerbated by obesity with excessive intramuscular fat deposition affecting muscle strength, quality, and contractility. ${ }^{43,45}$ MetS with sarcopenia have worse outcomes 
including weakness, functional impairment, increased morbidity, and mortality compared with either alone. ${ }^{46}$ The diagnosis of sarcopenia requires measurement of muscle strength where HGS is recommended. The participants in our study had no significant difference in HGS between the MetS and no-MetS groups. However, there were significant differences between the 2 groups when HGS was adjusted for BMI and BWT, respectively. HGS adjusted for BMI or BWT was a better predictor for MetS and its components than HGS alone. HGS adjusted for BMI or BWT revealed a significantly better dose-response relationship with adverse outcomes including ADL assistance and cognition. Our findings further support an earlier finding that adjusting muscle strength for BWT or BMI was a better predictor of functional performance, MetS, dyslipidemia, cardiovascular risks, or prediabetes. $^{14,15,47-49}$ There are various theories which require further validation in obese or overweight participants with MetS, including the altered oxidative capacity of muscle fibers, intramuscular fat accumulation, and muscle quality accounting for reduced muscle strength despite larger fat-free mass. $^{50}$

Our study has several strengths, including being a population-level study and including the old (70 to 79 years old) and old-old ( $\geq 80$ years old). Most studies on MetS are limited to $\leq 70$ years old. In addition to selfreported data, we have objective laboratory results to classify MetS and no-MetS. There are several limitations to our study. Firstly, participation in the health screening including blood tests was optional and those who did not agree to blood tests were younger, had lower cognition and education level, as well as a higher prevalence of hypertension; hence the prevalence of MetS may in fact be higher than observed. Secondly, the sample size of participants aged $\geq 80$ years was small, resulting in a wider $95 \%$ confidence interval. Thirdly, the higher prevalence of cognitive impairment in the MetS group could have affected recall and physical performance, including HGS and TUG. Lastly, our study did not collect sleep data including sleep quality and duration, which have been reported to be associated with MetS. ${ }^{51}$ More studies are needed to determine the prevalence and effect of MetS in the old-old and to identify biomarkers linking MetS to cancer, fatty liver, chronic diseases, and sarcopenia. In addition, further studies are needed to determine if interventions including resistance exercise and a high protein diet to improve muscle strength will lead to a lower prevalence of MetS.

\section{Conclusion}

The prevalence of MetS is on the rise in countries with aging population and it is associated with many negative outcomes including cardiovascular disease, functional decline, and cognitive impairment. Early detection of at-risk individuals is crucial as exercise programs have been shown to delay or reverse risks associated with MetS. HGS is a recommended assessment for muscle strength and diagnosis of sarcopenia. However, HGS adjusted for BWT or BMI is a better predictor for MetS, its components, and associated adverse outcomes. Further studies are needed prospectively to determine optimal cutoffs of HGS/weight and HGS/BMI to assess the risk of having MetS among community-dwelling older adults, if those with lower HGS/BMI are at greater risk of MetS and the effectiveness of interventions in averting the risk.

\section{Acknowledgment}

We thank the Saw Swee Hock School of Public Health, National University of Singapore in their collaboration.

\section{Disclosure}

The authors declare no conflicts of interest for this work.

\section{References}

1. Grundy SM. Metabolic syndrome pandemic. Arterioscler Thromb Vasc Biol. 2008;28(4):629-636. doi:10.1161/ATVBAHA.107.151092

2. Okamura T, Kokubo Y, Watanabe M, et al. A revised definition of the metabolic syndrome predicts coronary artery disease and ischemic stroke after adjusting for low density lipoprotein cholesterol in a 13-year cohort study of Japanese: the Suita study. Atherosclerosis. 2011;217(1):201-206. doi:10.1016/j.atherosclerosis.2011.03.010

3. Kumar R, Priyadarshi RN, Anand U. Non-alcoholic fatty liver disease: growing burden, adverse outcomes and associations. J Clin Transl Hepatol. 2020;8(1):76-86. doi:10.14218/JCTH.2019.00051

4. Cowie CC, Rust KF, Ford ES, et al. Full accounting of diabetes and pre-diabetes in the U.S. population in 1988-1994 and 2005-2006. Diabetes Care. 2009;32(2):287-294. doi:10.2337/dc08-1296

5. Gami AS, Witt BJ, Howard DE, et al. Metabolic syndrome and risk of incident cardiovascular events and death: a systematic review and meta-analysis of longitudinal studies. J Am Coll Cardiol. 2007;49 (4):403-414. doi:10.1016/j.jacc.2006.09.032

6. Kim G, Lee SE, Jun JE, et al. Increase in relative skeletal muscle mass over time and its inverse association with metabolic syndrome development: a 7-year retrospective cohort study. Cardiovasc Diabetol. 2018;17(1):23. doi:10.1186/s12933-018-0659-2

7. Kim G, Kim JH. Impact of skeletal muscle mass on metabolic health. Endocrinol Metab (Seoul). 2020;35(1):1-6. doi:10.3803/EnM.2020. 35.1 .1

8. Park SJ, Ryu SY, Park J, Choi SW. Association of sarcopenia with metabolic syndrome in Korean population using 2009-2010 Korea national health and nutrition examination survey. Metab Syndr Relat Disord. 2019;17(10):494-499. doi:10.1089/met.2019.0059 
9. Gale CR, Martyn CN, Cooper C, Sayer AA. Grip strength, body composition, and mortality. Int J Epidemiol. 2007;36(1):228-235. doi:10.1093/ije/dyl224

10. Sayer AA, Syddall HE, Martin HJ, Dennison EM, Roberts HC, Cooper C. Is grip strength associated with health-related quality of life? Findings from the Hertfordshire cohort study. Age Ageing. 2006;35(4):409-415. doi:10.1093/ageing/afl024

11. Wickham C, Cooper C, Margetts BM, Barker DJ. Muscle strength, activity, housing and the risk of falls in elderly people. Age Ageing 1989;18(1):47-51. doi:10.1093/ageing/18.1.47

12. Gubelmann C, Vollenweider P, Marques-Vidal P. Association of grip strength with cardiovascular risk markers. Eur J Prev Cardiol. 2017;24(5):514-521. doi:10.1177/2047487316680695

13. Hong S. Association of relative handgrip strength and metabolic syndrome in Korean older adults: Korea national health and nutrition examination survey VII-1. J Obes Metab Syndr. 2019;28(1):53-60. doi:10.7570/jomes.2019.28.1.53

14. Kim BM, Yi YH, Kim YJ, et al. Association between relative handgrip strength and dyslipidemia in Korean adults: findings of the 2014-2015 Korea national health and nutrition examination survey. Korean J Fam Med. 2020.

15. Ploutz-Snyder LL, Manini T, Ploutz-Snyder RJ, Wolf DA. Functionally relevant thresholds of quadriceps femoris strength. J Gerontol a Biol Sci Med Sci. 2002;57(4):B144-152. doi:10.1093/gerona/57.4.B144

16. Barbat-Artigas S, Rolland Y, Zamboni M, Aubertin-Leheudre M. How to assess functional status: a new muscle quality index. $J$ Nutr Health Aging. 2012;16(1):67-77. doi:10.1007/s12603-012-0004-5

17. Merchant RA, Chen MZ, Tan LWL, Lim MY, Ho HK, van Dam RM. Singapore healthy older people everyday (HOPE) study: prevalence of frailty and associated factors in older adults. J Am Med Dir Assoc. 2017;18(8):734.e9-734.e14. doi:10.1016/j.jamda.2017.04.020

18. Merchant RA, van Dam RM, Tan LWL, Lim MY, Low JL, Morley JE. Vitamin D binding protein and vitamin D levels in multi-ethnic population. J Nutr Health Aging. 2018;22 (9):1060-1065. doi:10.1007/s12603-018-1114-5

19. Folstein MF, Folstein SE, McHugh PR. "Mini-mental state". A practical method for grading the cognitive state of patients for the clinician. J Psychiatr Res. 1975;12(3):189-198. doi:10.1016/00 22-3956(75)90026-6

20. Sahadevan S, Lim P, Tan NJL, Chan SP. Diagnostic performance of two mental status tests in the older Chinese: influence of education and age on cut-off values. Int J Geriatr Psychitr. 2000;15(3):234-241.

21. Fuentes-García A. Katz Activities of Daily Living Scale. In: Michalos AC, editor. Encyclopedia of Quality of Life and WellBeing Research. Dordrecht: Springer Netherlands; 2014:3465-3468.

22. Diaz de Leon Gonzalez E, Gutierrez Hermosillo H, Martinez Beltran JA, et al. Validation of the FRAIL scale in Mexican elderly: results from the Mexican health and aging study. Aging Clin Exp Res. 2016;28(5):901-908. doi:10.1007/s40520-015-0497-y

23. Woo J, Yu R, Wong M, Yeung F, Wong M, Lum C. Frailty screening in the community using the FRAIL scale. J Am Med Dir Assoc. 2015;16(5):412-419. doi:10.1016/j.jamda.2015.01.087

24. Gusi N, Olivares PR, Rajendram R. The EQ-5D health-related quality of life questionnaire. In: Preedy VR, Watson RR, editors. Handbook of Disease Burdens and Quality of Life Measures. New York: Springer New York; 2010:87-99.

25. Podsiadlo D, Richardson S. The timed "Up \& Go": a test of basic functional mobility for frail elderly persons. $J$ Am Geriatr Soc. 1991;39(2):142-148. doi:10.1111/j.1532-5415.1991.tb01616.x

26. Arai H, Yamamoto A, Matsuzawa Y, et al. Prevalence of the metabolic syndrome in elderly and middle-aged Japanese. J Clin Gerontol Geriatr. 2010;1(2):42-47. doi:10.1016/j.jcgg.2010.10.011

27. Ko GT, Cockram CS, Chow CC, et al. High prevalence of metabolic syndrome in Hong Kong Chinese-comparison of three diagnostic criteria. Diabetes Res Clin Pract. 2005;69(2):160-168. doi:10.1016/ j.diabres.2004.11.015
28. Strath S, Swartz A, Parker S, Miller N, Cieslik L. Walking and metabolic syndrome in older adults. J Phys Act Health. 2007;4 (4):397-410. doi:10.1123/jpah.4.4.398

29. Mankowski RT, Aubertin-Leheudre M, Beavers DP, et al. Sedentary time is associated with the metabolic syndrome in older adults with mobility limitations-the LIFE study. Exp Gerontol. 2015;70:32-36. doi:10.1016/j.exger.2015.06.018

30. Denys K, Cankurtaran M, Janssens W, Petrovic M. Metabolic syndrome in the elderly: an overview of the evidence. Acta Clin Belg. 2009;64(1):23-34. doi:10.1179/acb.2009.006

31. Lee EG, Choi JH, Kim KE, Kim JH. Effects of a walking program on self-management and risk factors of metabolic syndrome in older Korean adults. J Phys Ther Sci. 2014;26(1):105-109. doi:10.1589/ jpts.26.105

32. Kemmler W, Von Stengel S, Engelke K, Kalender WA. Exercise decreases the risk of metabolic syndrome in elderly females. Med Sci Sports Exerc. 2009;41(2):297-305. doi:10.1249/MSS.0b013e318 $18844 \mathrm{~b} 7$

33. Yu W, Hu X, Yang L, et al. Duration of reproductive years and time since menopause were associated with metabolic syndrome in postmenopausal parous women of Chinese ancestry. Menopause. 2020;27 (2):216-222. doi:10.1097/GME.0000000000001445

34. Inaraja V, Thuissard I, Andreu-Vazquez C, Jodar E. Lipid profile changes during the menopausal transition. Menopause. 2020; Publish Ahead of Print. doi:10.1097/GME.0000000000001532

35. Chua KY, Lim WS, Lin X, Yuan JM, Koh WP. Handgrip strength and timed up-and-go (TUG) test are predictors of short-term mortality among elderly in a population-based cohort in Singapore. J Nutr Health Aging. 2020;24(4):371-378. doi:10.1007/s12603-020-1337-0

36. Liu CY, Zhou HD, Xu ZQ, Zhang WW, Li XY, Zhao J. Metabolic syndrome and cognitive impairment amongst elderly people in Chinese population: a cross-sectional study. Eur J Neurol. 2009;16 (9):1022-1027. doi:10.1111/j.1468-1331.2009.02640.x

37. Chode S, Malmstrom TK, Miller DK, Morley JE. Frailty, diabetes, and mortality in middle-aged African Americans. J Nutr Health Aging. 2016;20(8):854-859. doi:10.1007/s12603-016-0801-3

38. Farr SA, Yamada KA, Butterfield DA, et al. Obesity and hypertriglyceridemia produce cognitive impairment. Endocrinology. 2008;149 (5):2628-2636. doi:10.1210/en.2007-1722

39. Morley JE. Diabetes: the diabetic brain. Nat Rev Endocrinol. 2017;13 (10):570-571. doi:10.1038/nrendo.2017.111

40. Kwon HM, Kim BJ, Park JH, et al. Significant association of metabolic syndrome with silent brain infarction in elderly people. J Neurol. 2009;256(11):1825-1831. doi:10.1007/s00415-009-5201-8

41. Park SW, Goodpaster BH, Lee JS, et al. Excessive loss of skeletal muscle mass in older adults with type 2 diabetes. Diabetes Care. 2009;32(11):1993-1997. doi:10.2337/dc09-0264

42. Sarparanta J, Garcia-Macia M, Singh R. Autophagy and mitochondria in obesity and type 2 diabetes. Curr Diabetes Rev. 2017;13 (4):352-369. doi:10.2174/1573399812666160217122530

43. Rubio-Ruiz ME, Guarner-Lans V, Perez-Torres I, Soto ME. Mechanisms underlying metabolic syndrome-related sarcopenia and possible therapeutic measures. Int J Mol Sci. 2019;20(3):647. doi:10.3390/ijms20030647

44. Mesinovic J, McMillan LB, Shore-Lorenti C, De Courten B, Ebeling PR, Scott D. Metabolic syndrome and its associations with components of sarcopenia in overweight and obese older adults. J Clin Med. 2019;8(2):145. doi:10.3390/jcm8020145

45. Kalinkovich A, Livshits G. Sarcopenic obesity or obese sarcopenia: a cross talk between age-associated adipose tissue and skeletal muscle inflammation as a main mechanism of the pathogenesis. Ageing Res Rev. 2017;35:200-221. doi:10.1016/j.arr.2016.09.008

46. Everson-Rose SA, Paudel M, Taylor BC, et al. Metabolic syndrome and physical performance in elderly men: the osteoporotic fractures in men study. J Am Geriatr Soc. 2011;59(8):1376-1384. doi:10.1111/ j.1532-5415.2011.03518.x 
47. Manda CM, Hokimoto T, Okura T, Isoda H, Shimano H, Wagatsuma Y. Handgrip strength predicts new prediabetes cases among adults: a prospective cohort study. Prev Med Rep. 2020;17:101056. doi:10. 1016/j.pmedr.2020.101056

48. Peterson MD, Duchowny K, Meng Q, Wang Y, Chen X, Zhao Y. Low normalized grip strength is a biomarker for cardiometabolic disease and physical disabilities among U.S. and Chinese adults. J Gerontol a Biol Sci Med Sci. 2017;72(11):1525-1531. doi:10.1093/gerona/glx031

49. Kawamoto R, Ninomiya D, Kasai Y, et al. Handgrip strength is associated with metabolic syndrome among middle-aged and elderly community-dwelling persons. Clin Exp Hypertens. 2016;38 (2):245-251. doi:10.3109/10641963.2015.1081232
50. Lagace JC, Brochu M, Dionne IJ. A counterintuitive perspective for the role of fat-free mass in metabolic health. J Cachexia Sarcopenia Muscle. 2020;11(2):343-347. doi:10.1002/jcsm.12520

51. Lu K, Zhao Y, Chen J, Hu D, Xiao H. Interactive association of sleep duration and sleep quality with the prevalence of metabolic syndrome in adult Chinese males. Exp Ther Med. 2020;19(2):841-848. doi:10.3892/etm.2019.8290

Diabetes, Metabolic Syndrome and Obesity: Targets and Therapy

\section{Publish your work in this journal}

Diabetes, Metabolic Syndrome and Obesity: Targets and Therapy is an international, peer-reviewed open-access journal committed to the rapid publication of the latest laboratory and clinical findings in the fields of diabetes, metabolic syndrome and obesity research. Original research, review, case reports, hypothesis formation, expert opinion and commentaries are all considered for publication. The manuscript management system is completely online and includes a very quick and fair peer-review system, which is all easy to use. Visit http://www.dovepress.com/testimonials.php to read real quotes from published authors. 